\title{
Transferrin changes in haemodialysed patients
}

\author{
Dorota Formanowicz • Piotr Formanowicz
}

Received: 12 August 2010/Accepted: 11 March 2011/Published online: 1 April 2011

(C) The Author(s) 2011. This article is published with open access at Springerlink.com

\begin{abstract}
Transferrin (Tf) is a glycoprotein responsible for iron transport in the human body. Physiologically in reaction with Concanavalin $\mathrm{A}$, Tf occurs in four distinct variants Tf1, Tf2, Tf3 (apo-Tf) and Tf4. It was reported recently that $\mathrm{Tf}$ is changing, particularly during acute phase response, taking place among others in end-stage renal disease. In this study, we wanted to find the answer to three main questions: firstly, how $\mathrm{Tf}$ is changing in patients treated with maintenance haemodialysis (mHD), secondly, whether there are any $\mathrm{Tf}$ changes in the course of $\mathrm{mHD}$ treatment, and thirdly, what factors can affect $\mathrm{Tf}$ microheterogeneity in these patients. Studies were performed on 80 haemodialysed patients and 21 healthy volunteers. The Tf concentration was determined by the rocket immunoelectrophoresis, and its microheterogeneity was assessed by the ConA crossed immunoaffinity electrophoresis. During the annual
\end{abstract}

\footnotetext{
D. Formanowicz $(\square)$

Department of Clinical Biochemistry, Poznan University of Medical Sciences, Grunwaldzka 6, 60-780 Poznan, Poland

e-mail: doforman@ump.edu.pl

P. Formanowicz

Institute of Computing Science, Poznan University

of Technology, Piotrowo 2, 60-965 Poznan, Poland

P. Formanowicz

Institute of Bioorganic Chemistry, Polish Academy

of Sciences, Noskowskiego 12/14, 61-704 Poznan, Poland
}

observation of the distribution of the Tf variants, we have found both changes of the percentage contents of all $\mathrm{Tf}$ variants in the whole $\mathrm{Tf}$ concentration and a significant decrease in Tf2, Tf3 and Tf4 serum concentrations. Moreover, we found that decrease in the renal function, duration of $\mathrm{mHD}$, and inflammation may contribute to these above-mentioned changes, which are probably the factors that should be taken into account when explaining the mechanisms of persistence of anaemia in haemodialysed patients.

Keywords Anaemia - Haemodialysis . Microheterogeneity · Transferrin variants · Iron

\section{Introduction}

Anaemia is very common in patients with chronic kidney disease (CKD). Factors likely contributing to anaemia in CKD include blood loss, shortened red cell life span, vitamin deficiencies, the "uremic milieu" (a term that attempts to explain the multiple organ dysfunction of CKD), erythropoietin (EPO) deficiency, iron deficiency, and inflammation. Much is known about the participation of the abovementioned factors in anaemia, but this knowledge is still incomplete. One of the not-yet-fully-explained disturbances is that of iron balance in CKD. The process of human body iron homoeostasis is a very complex issue, and its main part was described in 
detail by Formanowicz et al. [1], Sackmann et al. [2], and Formanowicz et al. [3].

Not fully understood and explained phenomenon of persistent anaemia and especially unexplained iron balance disturbances in patients with CKD has become the reason for the design of this study.

It should be noticed that, in CKD, the ability to provide enough iron for erythropoiesis is disrupted at several points in the body iron cycle. One of these points is iron transport. The protein responsible for this transport is transferrin (Tf). For reasons not yet fully explained, Tf levels in CKD are one-half to onethird of normal levels, diminishing the capacity of the iron-transporting system. This situation is then aggravated by the well-known inability to release stored iron from macrophages, scavenging iron from senescent red blood cells and from hepatocytes in CKD [4].

There are two main reasons for which this study focused on the changes in Tf in CKD. First, we know little about these changes in CKD. Second, in CKD several factors exist, like inflammatory process and malnutrition, which may contribute to the $\mathrm{Tf}$ changes.

In this part of the work, to understand the qualitative changes of $\mathrm{Tf}$, its chemical structure and variability will be presented. Tf $(79.57 \mathrm{kDa})$ consists of three substructural domains: a single polypeptide chain, arranged in two lobes, representing the $\mathrm{N}$ terminal and C-terminal halves of the molecule [5], two independent, structurally similar but functionally distinct iron-binding sites and usually two $N$-linked complex glycan chains. It should be noticed that the oligosaccharide chains are usually composed of a common pentasaccharide core and various quantity of sialic acid residues, galactose and/or fucose molecules and $\mathrm{N}$-acetylglucosamine, creating the antennary chains. They differ also in the degree of branching (antennarity), where to the most basic biantennary structure additional antenna may be bound, producing tri-, tetra- or even more antennary glycans $[6,7]$.

A schematic representation of the transferrin molecule is shown in Fig. 1.

The heterogeneity of $\mathrm{N}$-glycan chains, varying iron load (depending on iron supply) and modification of the polypeptide chain ( $\mathrm{Tf}$ variants) create a distinct $\mathrm{Tf}$ microheterogeneity [8]. If the Tf molecules contain oligosaccharides of different structures, this phenomenon is known as a "major microheterogeneity" [9]; in turn, the difference in the quantity and quality of (a)

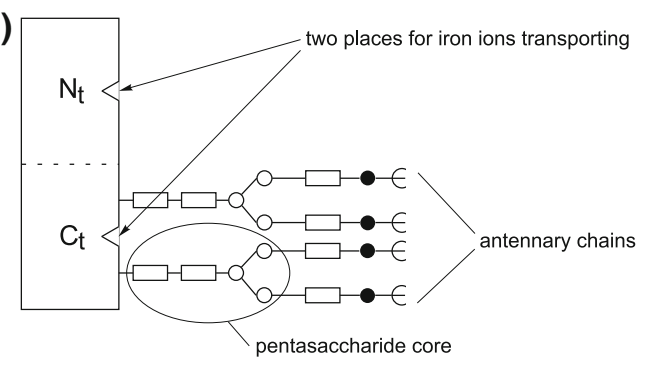

(b)

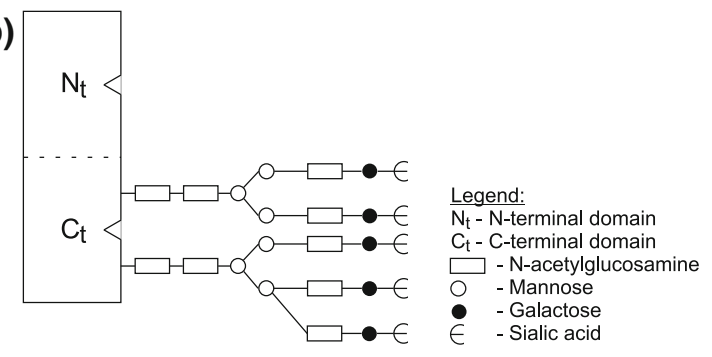

Fig. 1 Schematic representation of the transferrin molecule. (a) Tetra-sialo-Tf while in (b) penta-sialo-Tf are shown

the oligosaccharides residues is qualified as the "marginal microheterogeneity" [10].

In many physiological and pathological conditions, like stress, pregnancy [11, 12], alcoholism [6], liver failure in alcoholics [13] the changes in the number of $\mathrm{Tf}$ variants, changes in their proportions, and the presence of completely new variants have been revealed. All of these studies emphasized the important influence of the inflammatory process and oxidative stress on these changes.

Despite numerous studies on the variability of Tf, little is known about the importance of each of its variants. The results obtained from few previously conducted studies are inconclusive. For humans, an increase in affinity of Tf receptors for higher branched glycans, was reported by Rudolph [14], but could not be confirmed by other authors, like Léger et al. [11] and van Dijk et al. [15]. It should be emphasized that the binding of transferrin to its receptor on the cell surface is crucial in iron metabolism, because it allows the transfer of iron directly into the cell.

Although we have little information about the participation of individual variations in the transport of iron, changes in their proportions seem to be important in maintaining the iron disturbances CKD. Only few studies have been conducted on this topic so far. In our preliminary studies [16, 17, 18, 19, 20], we tried to find the answer to one main question 
of whether the microheterogeneity profile of $\mathrm{Tf}$ is changing in CKD, particularly in haemodialysed patients, in whom these changes could be particularly relevant. Based on the results of these studies, it can be concluded that $\mathrm{Tf}$ is changing both quantitatively and qualitatively in haemodialysed patients. It was revealed that the sera concentrations of three of the four variants, i.e. Tf1, Tf2 and Tf4, increased, while the Tf3 variant decreased, when compared to healthy volunteers as controls. Moreover, the proportion of those variants in the whole Tf differed significantly between studied patients and controls. The limitation of these studies was the small sample size. Therefore, in the present study we have focused on a thorough analysis of the $\mathrm{Tf}$ microheterogeneity phenomenon in a larger group of haemodialysed patients. On the other hand, we wanted to find the answer to two other questions: first, whether there are any changes in the Tf in a course of 1 year of maintenance haemodialysis treatment (mHD), and secondly, what factors can affect Tf microheterogeneity in these patients. To the best of our knowledge, results from such analyses have not been published so far.

\section{Materials and methods}

Patients and healthy controls subjects

This study was carried out on a group of 101 subjects, i.e. 80 patients (mHD group) from the Department of Nephrology, Transplantology and Internal Medicine, at Poznań University of Medical Sciences, enrolled to the haemodialysis treatment, according to The National Kidney Foundation Kidney Disease Outcomes Quality Initiative (NKF KDOQI ${ }^{\mathrm{TM}}$ ) guidelines [21] on the basis of their estimated glomerular filtration rate (eGFR) — calculated by the 4-variable MDRD Study equation, i.e. serum creatinine, age, race, and gender [22], and 21 healthy volunteers as controls (HV group). Patients were examined twice, i.e. at the beginning (mHD1) and after 1 year of the study (mHD2). For every examined person the demographic data, including age and gender were recorded. Additionally, from all haemodialysed patients, clinical data, like eGFR, kidney disease diagnosis, comorbid conditions, current treatments, i.e. the doses of rHuEPO and iron, the duration of end-stage renal disease (ESRD) before enrolling to HD (months) and the duration of HD treatment (months) were collected.

Haemodialysis sessions were performed three times a week, for $4 \mathrm{~h}$ each, with polysulphone capillary dialysers, to achieve dialysis adequacy $\mathrm{K} t$ / $V>1.2$.

After HD sessions, depending on the individual patient's clinical status and EPO responsiveness, patients received intravenous erythropoietin alpha $\left[\right.$ Eprex $\left.^{\circledR}\right]$. Furthermore, undiluted iron sucrose-ferric hydroxide saccharin $\left[\right.$ Venofer $\left.{ }^{\circledR}\right]$ ) treatment in addition to EPO was administered according to NKF $\mathrm{KDOQI}^{\mathrm{TM}}$ guidelines. Intravenous iron was prescribed in slow injection $(\leq 20 \mathrm{mg} / \mathrm{min})$ given directly into the dialysis line. The goal of administering iron sucrose was to maintain TSAT between 30 and $45 \%$, serum ferritin between 200 and $800 \mathrm{ng} / \mathrm{ml}$, $\mathrm{Hb}$ between 11 and $12.5 \mathrm{~g} / \mathrm{dl}$, and Hct between 33 and $36 \%$.

We included in the study only those mHD patients who met the following criteria: a stable clinical state, no recent thrombosis or acute infection, absence of inflammation, i.e. normal serum concentration of C-reactive protein, interleukin-1 and interleukin-6 and TNF-alpha, no comorbid iron metabolism disturbances at present, i.e. high serum ferritin and low serum iron level, without uncontrolled hypertension, no oral contraception in women of child-bearing age, stable and no more than twice the normal alanine aminotransferase and aspartate aminotransferase levels (upper range $45 \mathrm{U} / \mathrm{l}$ ). None of the patients investigated had received blood transfusions during the previous 1.5 months.

The control group (HV) consisted of 21 age- and sex-matched healthy volunteers without any laboratory and clinical symptoms of anemia or inflammation. All of them had normal renal function, estimated by urinalysis, creatinine serum concentration and eGFR.

The characteristics of the studied groups are shown in Table 1.

Design of the study

Approval was obtained from the local ethics committee before the start of the study, and all participants gave a written informed consent. 
Table 1 General characteristics of the studied groups

\begin{tabular}{|c|c|c|c|}
\hline \multirow[t]{2}{*}{ Studied groups } & \multicolumn{2}{|l|}{$\mathrm{mHD}$} & \multirow[t]{2}{*}{$\mathrm{HV}$ as controls } \\
\hline & mHD1 & mHD2 & \\
\hline Number of studied subjects (men/women) & $50 / 30$ & & $13 / 8$ \\
\hline Age (years) & $55.6 \pm 14$ & $56.6 \pm 14$ & $54.8 \pm 16$ \\
\hline eGFR $\left(\mathrm{kg} / \mathrm{min} / 1.73 \mathrm{~m}^{2}\right)$ & $5.5 \pm 6.9$ & $4.31 \pm 2.9$ & $115.6 \pm 31.9$ \\
\hline \multirow[t]{3}{*}{ Diagnosis (number of subjects) } & \multicolumn{2}{|c|}{ Glomerulonephritis (40) } & - \\
\hline & \multicolumn{2}{|c|}{ Diabetic nephropathy (31) } & \\
\hline & \multicolumn{2}{|c|}{ Hypertensive nephrosclerosis (9) } & \\
\hline Number of subjects with cardiovascular disease & 64 & & - \\
\hline Number of subjects supplemented with rHuEPO & 70 & 74 & - \\
\hline Weekly doses of rHuEPO [IU] (mean dose among patients getting rHuEPO) & $4039 \pm 2592$ & $4260 \pm 2847$ & - \\
\hline Number of subjects supplemented with iron & 65 & 69 & - \\
\hline Weekly doses of iron [mg] (mean dose among patients getting iron) & $41.05 \pm 63.758$ & $106 \pm 84.297$ & - \\
\hline Time of ESRD before enrolling to HD (months) & $19 \pm 2$ & & - \\
\hline Time of HD treatment (months) & $46 \pm 6$ & $58 \pm 6$ & - \\
\hline
\end{tabular}

Subjects were divided into patients treated with maintenance haemodialysis (mHD1—at the beginning of the study, mHD2-after 1 year of the study) and healthy volunteers (HV) as controls

Results were given as mean $\pm \mathrm{SD}$

eGFR estimated glomerular filtration rate, $r H u E P O$ recombinant human erythropoietin, ESRD end-stage renal disease, HD haemodialysis

The starting time of the study (baseline) for each patient and control was defined when first blood sample was drawn. Blood samples were drawn from mHD patients before a midweek dialysis session (before heparin administration), at the same time as their usual monitoring blood tests were performed, and after the dialysis session, from the arterial line of the haemodialysis system, immediately before the disconnection of the extracorporeal circulation (only for urea concentration, necessary for standardised $K t / V$, a marker of adequacy of dialysis determination). Standard Kt/V was measured according to Gotch and Sargent formula [23]. Blood was drawn without stasis.

All patients and healthy volunteers underwent a thorough physical examination. The following anthropometrical data were determined: weight, height, body mass index (BMI) and waist-to-hip ratio (WHR) (see in Table 3). In all subjects, the selected serum parameters (detailed further), including selected haematological parameters, proteins and cytokines, lipid profile, and liver function tests at baseline and after one year follow-up were recorded. In haemodialysed patients, serum ferritin and serum iron were assessed 1 week following administration of intravenous iron.
Blood samples were taken within the same period in fasting patients and in fasting control subjects. The sera were immediately separated by centrifugation after clotting and frozen at $-80^{\circ} \mathrm{C}$ until analysis. All samples were run in duplicate. They were stored and processed in an identical fashion. All tests were performed according to the manufacturer's instructions.

The methodology used for the laboratory tests was as follows:

Analysed parameters used for characteristics of the studied groups

\section{Selected haematological parameters}

The red blood cell count (RBC) $\left[10^{12} / 1\right]$, haematocrit (HCT) [\%], haemoglobin (HGB) [g/dl], mean corpuscular haemoglobin $(\mathrm{MCH})[\mathrm{pg}]$ and mean corpuscular volume (MCV) [fl] were estimated by a conductometry method, using haematological analyser (Sysmex K-4500, ICN).

The values of the total iron-binding capacity (TIBC) $[\mu \mathrm{mol} / \mathrm{l}]$, unsaturated iron-binding capacity (UIBC) $[\mu \mathrm{mol} / \mathrm{l}]$ and serum iron concentration (Fe) $[\mu \mathrm{mol} / \mathrm{l}]$ were evaluated by a colorimetric method 
with ferrozin, using biochemical analyser (Cobas Integra, Roche).

The saturation of transferrin (TSAT) [\%] was calculated as a ratio of the serum iron concentration to the value of the TIBC.

Serum ferritin $(\mathrm{sFn})[\mathrm{ng} / \mathrm{mL}]$ was estimated by a chemiluminescence method with the mice monoclonal antibodies specific to the human ferritin, using immunochemical analyser (Elecsys 2010, Roche).

The serum concentrations of the selected haematological parameters used for characteristics of the studied groups are presented in Table 2.

\section{Selected proteins and cytokines}

For the quantitative determination of serum C-reactive protein $(\mathrm{CRP})[\mathrm{mg} / \mathrm{l}]-\mathrm{N}$ high sensitivity CRP reagent ( $h s C R P$ ) - , an in vitro diagnostic assay consisting of a suspension of polystyrene particles coated with the mouse monoclonal antibodies to CRP and nephelometer (DADE Behring Analyzer II) were used.

The sera interleukin 6 (IL-6), interleukin 1 (IL-1) and tumour necrosis factor alpha (TNF- $\alpha$ ) $[\mathrm{pg} / \mathrm{ml}]$ concentrations were evaluated by an enzyme-linked immunosorbent assay (ELISA) method using (R\&D Systems) Quantikine HS human IL-6 kit, IL-1 kit and TNF kit, respectively.

\section{Selected liver metabolism parameters}

The serum bilirubin concentration $(\mathrm{mg} / \mathrm{dl})$ was assessed by colorimetric method by using Cobas Integra Analyser (Roche).

The activities of the aminotransferases, the alanine aminotransferase (ALT) [IU/l] and the aspartate aminotransferase (AST) [IU/l], were measured by kinetic method using Cobas Integra Analyser (Roche).

The concentration of albumin was determined by immunonephelometric, using a set of $\mathrm{N}$-Antiserum to

Table 2 Selected haematological parameters used for characteristics of the studied groups

\begin{tabular}{|c|c|c|c|c|}
\hline \multirow[t]{2}{*}{ Parameter } & \multicolumn{2}{|l|}{$\mathrm{mHD}$} & \multirow{2}{*}{$\begin{array}{l}P \text { value } \\
\text { mHD1 vs. mHD2 }\end{array}$} & \multirow[t]{2}{*}{$\mathrm{HV}$ as controls } \\
\hline & mHD1 & mHD2 & & \\
\hline $\operatorname{RBC}\left(10^{12} / 1\right)$ & $3.25 \pm 0.47 * * *$ & $3.11 \pm 0.42 * * *$ & 0.03 & $4.6 \pm 0.8$ \\
\hline $\operatorname{HCT}(\%)$ & $33.3 \pm 318 * * *$ & $32.31 \pm 3.9 * * *$ & 0.64 & $44.3 \pm 7.2$ \\
\hline HGB (g/dl) & $10.62 \pm 1.54 * * *$ & $10.5 \pm 1.21 * * *$ & 0.76 & $14.9 \pm 2.0$ \\
\hline $\mathrm{MCH}(\mathrm{pg})$ & $32.86 \pm 4.79$ & $34.16 \pm 3.7$ & 0.15 & $34.2 \pm 3.7$ \\
\hline MCV (fL) & $98.68 \pm 5.86 * * *$ & $98.91 \pm 5.72 * * *$ & 0.81 & $104.6 \pm 11.1$ \\
\hline TIBC $(\mu \mathrm{mol} / \mathrm{l})$ & $37.66 \pm 5.27 * * *$ & $42.4 .8 \pm 8.47 * * *$ & 0.0001 & $59.6 \pm 5.3$ \\
\hline UIBC $(\mu \mathrm{mol} / 1)$ & $21.92 \pm 8.04 * * *$ & $17.942 \pm 8.2 * * *$ & 0.0021 & $36.4 \pm 3.1$ \\
\hline $\mathrm{Fe}(\mu \mathrm{mol} / \mathrm{l})$ & $15.73 \pm 6.68 * * *$ & $15.06 \pm 7.86 * * *$ & 0.63 & $22.7 \pm 9.1$ \\
\hline sFn (ng/ml) & $1749.55 \pm 179.98 * * *$ & $1804.72 \pm 1086.53 * * *$ & 0.88 & $188.2 \pm 10.6$ \\
\hline TSAT $(\%)$ & $42.6 \pm 18.95 * * *$ & $37.84 .8 \pm 24.7 * * *$ & 0.21 & $38.4 \pm 6.5$ \\
\hline
\end{tabular}

Subjects were divided into patients treated with maintenance haemodialysis (mHD1—at the beginning of the study, mHD2-after 1 year of the study) and healthy volunteers (HV) as controls

The differences between mHD1 and mHD2 were estimated by paired $t$ test. To compare mHD1 and mHD2 with HV as controls, unpaired the Student's $t$ test for normally distributed variables and the Mann-Whitney rank sum test for the variables with nonnormal distribution were used

Data were given are means $\pm \mathrm{SD}$

$m H D 1$ patients treated with maintenance haemodialysis at the beginning of the study, $m H D 2$ patients treated with maintenance haemodialysis after 1 year of the study, $R B C$ red blood cells, $H C T$ haematocrit, $H G B$ haemoglobin, $M C H$ mean haemoglobin concentration, $M C V$ mean corpuscular volume, $T I B C$ total iron-binding capacity, UIBC unsaturated iron-binding capacity, $F e$ serum iron, $s F n$ serum ferritin, TSAT transferrin saturation

Significant differences when $P<0.05$

*** $P<0.001$ versus $\mathrm{HV}$ 
Human Albumin from Dade Behring, USA, and nephelometer Dade Behring Analyzer II.

\section{Selected lipids metabolism parameters}

Total cholesterol and triglycerides were determined by colorimetric method with glycerol phosphate oxidase and 4-aminoantipyrine (GPO/PAP), using a biochemical analyzer Cobas Integra 400, Roche. Results were expressed in units of mmol/l.

The serum concentrations of the analysed proteins and cytokines, liver metabolism parameters and lipid parameters used for characteristics of the studied groups are shown in Table 3.

\section{Laboratory determinations}

In every patient and control, apart from parameters that became put-upon to the characteristics of the examined groups, transferrin serum concentrations and transferrin microheterogeneity were examined.

\section{Transferrin concentration}

To measure transferrin serum concentration $(\mathrm{mg} / \mathrm{l})$, the rocket immunoelectrophoresis according to Laurell [24] was used. The $1 \%$ agarose (Prona) in Tris-HCl buffer, $\mathrm{pH} 8.6$, was boiled and then cooled down to $56^{\circ} \mathrm{C}$. Agarose contained $40 \mu \mathrm{l}$ rabbit antihuman transferrin (Daktopatts). Then, it was cast on the plates. Electrophoresis was performed with $3 \mu \mathrm{l}$ sample serum (10-fold dilutions) and standard human serum (Daktopatts) (10- and 5-fold dilutions) at $1.5 \mathrm{~V} / \mathrm{cm}$ for $8 \mathrm{~h}$. Afterwards the plates were pressed, dried and stained with Coomassie Brilliant Blue R-250. Total serum concentrations of $\mathrm{Tf}$ were counted by using a planimetrical method. The areas of precipitations were proportional to the quantity of the antigen, i.e. Tf.

Table 3 Anthropometrical parameters, liver metabolism parameters and inflammatory markers in mHD group (mHD1-at the beginning of the study, mHD2 - after 1 year of the study) and in healthy volunteers (HV) as controls

\begin{tabular}{|c|c|c|c|c|}
\hline \multirow[t]{2}{*}{ Parameter } & \multicolumn{2}{|l|}{$\mathrm{mHD}$} & \multirow{2}{*}{$\begin{array}{l}P \text { value } \\
\text { mHD1 vs. mHD2 }\end{array}$} & \multirow[t]{2}{*}{$\mathrm{HV}$ as controls } \\
\hline & mHD1 & mHD2 & & \\
\hline WHR & $0.95 \pm 0.22$ & $0.94 \pm 0.12$ & 0.86 & $0.97 \pm 0.05$ \\
\hline BMI $\left(\mathrm{kg} / \mathrm{m}^{2}\right)$ & $25.16 \pm 4.69$ & $24.56 \pm 4.75$ & 0.07 & $25.6 \pm 4.3$ \\
\hline Cholesterol (mg/dl) & $178.9 \pm 47.4$ & $173 \pm 36.6$ & 0.49 & $170.8 \pm 24.3$ \\
\hline Triglycerides (mg/dl) & $179 \pm 22.6^{* *}$ & $175.7 \pm 23.2 * *$ & 0.54 & $92.3 \pm 26.5$ \\
\hline Albumin (g/l) & $38.091 \pm 5.34 * *$ & $36.73 \pm 4.12 * *$ & 0.19 & $44.7 \pm 1.71$ \\
\hline Bilirubin (mg/dl) & $0.6 \pm 0.22$ & $0.6 \pm 0.13$ & 0.91 & $0.6 \pm 0.1$ \\
\hline ALT (IU/l) & $15.34 \pm 7.8$ & $15.38 \pm 6.2$ & 0.83 & $15.57 \pm 7.4$ \\
\hline AST (IU/l) & $16.39 \pm 5.7$ & $16.44 \pm 7.42$ & 0.87 & $16.57 \pm 6.31$ \\
\hline hsCRP (mg/l) & $7.42 \pm 3.56^{* *}$ & $13.29 \pm 12.03 * * *$ & 0.00018 & $2.3 \pm 0.6$ \\
\hline IL-1 (pg/ml) & $3.98 \pm 0.67 * * *$ & $8.41 \pm 1.88 * * *$ & 0.00000 & $0.6 \pm 0.01$ \\
\hline IL-6 (pg/ml) & $36.82 \pm 39.84 * * *$ & $48.01 \pm 109.72 * * *$ & 0.5 & $4.3 \pm 0.7$ \\
\hline TNF-alpha (pg/ml) & $28.74 \pm 70.7 * * *$ & $25.57 \pm 21.10^{* * *}$ & 0.79 & $1.2 \pm 0.1$ \\
\hline
\end{tabular}

The differences between mHD1 and mHD2 were estimated by paired $t$ test. To compare mHD1 and mHD2 with HV as controls, unpaired the Student's $t$ test for normally distributed variables and the Mann-Whitney rank sum test for the variables with nonnormal distribution were used

Data were given are means $\pm \mathrm{SD}$

WHR waist-to-hip ratio, $B M I$ body mass index calculated as follows: $\mathrm{BMI}=$ weight $(\mathrm{kg}) /[\mathrm{height}(\mathrm{m})]^{2}, A L T$ alanine aminotransferase, $A S T$ aspartate aminotransferase, $h s C R P$ high sensitivity C-reactive protein, $I L-1$ interleukin $1, I L-6$ interleukin 6, TNF-alpha tumour necrosis factor-alpha

Significant differences when $P<0.05$

** $P<0.01 ; * * * P<0.001$ versus $\mathrm{HV}$ 


\section{Transferrin microheterogeneity}

Transferrin microheterogeneity was assessed by the crossed immunoaffinity electrophoresis according to Bog-Hansen [25]. The 1\% agarose (Prona, Spain) in Tris- $\mathrm{HCl}$ buffer, $\mathrm{pH} 8.6$, was prepared as mentioned earlier. The first dimension gel $(17.5 \mathrm{ml})$ contained $1.5 \mathrm{mg} / \mathrm{ml}$ lectin, i.e. Concanavalin A (ConA) as the diantennary-specific affinocomponent. Afterwards agarose was cast on the glass plates. Electrophoresis was performed with $10 \mu \mathrm{l}$ sample serum (10-fold dilutions in $0.9 \% \mathrm{NaCl}$ ) at $10 \mathrm{~V} / \mathrm{cm}$ and with Bromophenol Blue (BPB) mixed with albumin (marker of migration) for $2 \mathrm{~h}$. Next, a single slab, corresponding to migration of single sample serum, was submitted to the crossed immunoaffinity electrophoresis with anti-Tf. The second dimension gel (5.5 ml) contained $26 \mu \mathrm{l}$ rabbit anti-human transferrin (Daktopatts, Danmark) and a displacer, i.e. $1.5 \mathrm{mg} / \mathrm{ml}$ methyl $\alpha$-D-glucopyranoside (this sugar ousts the glycoprotein from her bond with the lectin and makes possible the migration of the protein in II direction). Electrophoresis was run at $1.5 \mathrm{~V} / \mathrm{cm}$ for $18 \mathrm{~h}$ on the second dimension plates, two slabs per plate. Then the plates were pressed, dried and stained with Coomassie Brilliant Blue R-250 and the relative occurrences of lectin-retarded and lectin-non-retarded glycoforms were calculated from the areas under the curves.

\section{Statistical analyses}

Statistical analysis was performed using Statistica 8.0 package. Data were expressed as mean values \pm SD (standard deviation) from 101 subjects. For testing the normality of variable distributions, the Shapiro-Wilk test was used. Non-parametrical tests were performed for the analysis of the non-normally distributed variables. For the comparison of variables obtained from patients at the beginning (mHD1) and after 1 year of the study (mHD2), paired $t$ test was used. To compare mHD1 and mHD2 with HV as controls, unpaired the Student's $t$ test for normally distributed variables and the Mann-Whitney rank sum test for the variables with non-normal distribution were used. To calculate the correlations between demographic data, selected variables, used form characteristics of the studied groups and the Tf variants, Spearman's rank correlation coefficient was used. The significance level was assumed to be equal to 0.05 .

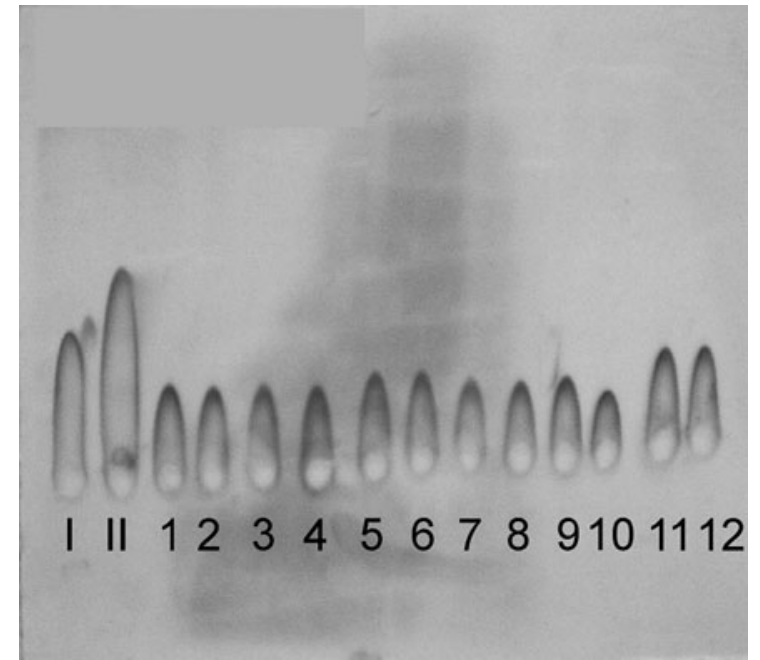

Fig. 2 The Tf patterns analysed by the rocket immunoelectrophoresis according to Laurell. (I-II) Standard solutions (controls); (1-6) sera samples from selected patients from mHD1group, (6-12) sera samples from selected patients from mHD2 group

\section{Results}

Analysis of the rocket immunoelectrophoresis patterns according to Laurell

The areas of precipitations proportional to the quantity of serum Tf showed the differences between mHD patients (the exemplary sera samples nos. 1-12) and healthy volunteers (the exemplary sera sample nos. I-II) (see Fig. 2).

After planimetrical analysis of the results, a considerable decrease in total serum $\mathrm{Tf}$ concentration in mHD group if compared to HV was found. The results summarised in Table 4 indicate a 2.5-fold decrease in the total amount of $\mathrm{Tf}$ between $\mathrm{mHD}$ patients (in both mHD1 and mHD2 groups) and HV group. However, no statistically significant changes in $\mathrm{Tf}$ were observed during 1 year of maintenance haemodialysis treatment, i.e. between mHD1 and mHD2 (see Table 4).

Analysis of the ConA crossed immunoaffinity electrophoresis patterns

The crossed immunoaffinity electrophoresis with ConA revealed four distinct $\mathrm{Tf}$ variants, i.e. Tf1, Tf2, Tf3 and Tf4 in the studied sera (see Figs. 3, 4). 
Table 4 The serum concentrations of total Tf were estimated by the rocket immunoelectrophoresis according to Laurell. After the obtainment of the precipitates, the results were counted on the basis on the planimetrical analysis of the surfaces of these precipitates

\begin{tabular}{|c|c|c|c|c|}
\hline \multirow[t]{2}{*}{ Parameter (mg/l) } & \multicolumn{2}{|l|}{$\mathrm{mHD}$} & \multirow{2}{*}{$\begin{array}{l}P \\
\text { mHD1 vs. mHD2 }\end{array}$} & \multirow[t]{2}{*}{$\mathrm{HV}$ as controls } \\
\hline & mHD1 & mHD2 & & \\
\hline Tf & $1408.27 \pm 410.98 * * *$ & $1386.486 \pm 426.726 * * *$ & 0.82 & $3532.123 \pm 761.31$ \\
\hline Tf1 & $132.035 \pm 63.17 *$ & $152.34 \pm 62.97$ & 0.19 & $150.12 \pm 51.1$ \\
\hline Tf2 & $227.412 \pm 76.56^{* * *}$ & $324.513 \pm 124.59$ & 0.0002 & $332.11 \pm 121.1$ \\
\hline Tf3 & $942.957 \pm 321.25^{* * *}$ & $802.21 \pm 401.31 * * *$ & 0.021 & $2882.91 \pm 721.3$ \\
\hline Tf4 & $150.865 \pm 60.70$ & $97.21 \pm 44.46^{* * *}$ & 0.00024 & $150.871 \pm 91.23$ \\
\hline
\end{tabular}

Subjects were divided into patients treated with maintenance haemodialysis (mHD1—at the beginning of the study, mHD2-after 1 year of the study) and in healthy volunteers (HV) as controls

The differences between mHD1 and mHD2 were estimated by paired $t$ test. To compare mHD1 and mHD2 with HV as controls, unpaired Student's $t$ test for normally distributed variables and the Mann-Whitney rank sum test for the variables with non-normal distribution were used

Tf-serum transferrin, Tf1-Tf4-Tf variants

Data were given are means $\pm \mathrm{SD}$

Significant differences when $P<0.05$

$* P<0.05$; *** $P<0.001$ versus $\mathrm{HV}$

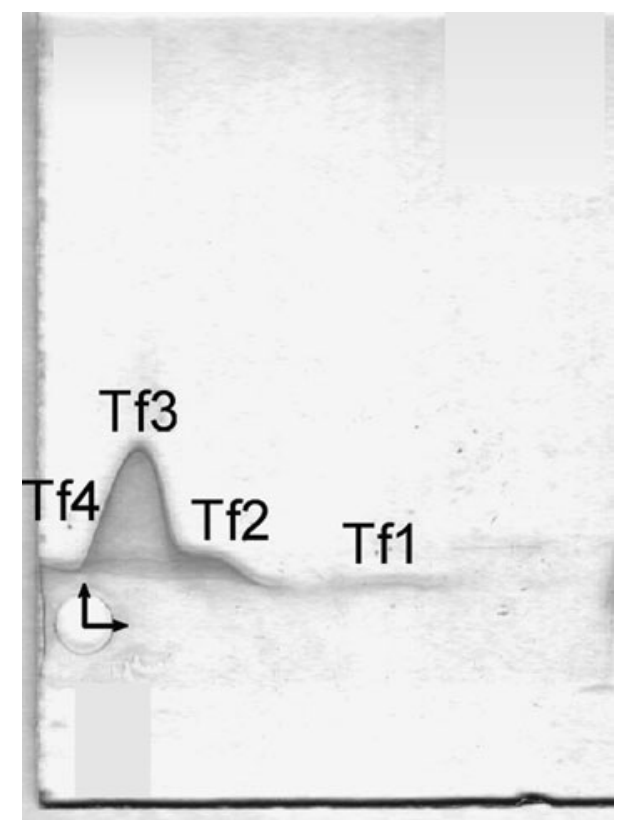

Fig. 3 Tf microheterogeneity patterns of serum of selected healthy volunteer analysed by the crossed affinoimmunoelectrophoresis according to Bog-Hansen in the presence of Concanavalin $\mathrm{A}$ in the first-dimension gel (the horizontal pointer) and rabbit anti-human transferrin in the seconddimension gel (the perpendicular pointer)

The results summarised in Table 4 indicate significant differences between $\mathrm{mHD}$ patients and $\mathrm{HV}$ group, particularly in the Tf3 serum concentration.
At the beginning of the study (mHD1), sera concentrations of Tf1, Tf 2 and Tf3 were significantly lower, in contrast to $\mathrm{Tf} 4$, if compared to HV group.

After 12 months of study, similar changes, except for changes of Tf1 were observed. It should be mentioned here that the changes in Tf 2 and Tf 3 has intensified. In addition, there was statistically significant decrease in the serum concentration of $\mathrm{Tf} 4$, which was not observed previously.

The per cent distribution of Tf variants in the whole serum Tf differed between studied groups, see on Fig. 5. In mHD groups, the percentage contents of Tf1, $\mathrm{Tf} 2$ and Tf4 variants in the total serum Tf were higher than in HV, contrary to Tf3 variant, whose percentage content decreased if compared to HV. Furthermore, interesting seems to be the decrease in the percentage content of Tf 3 variant in the total serum Tf observed in haemodialysed patients during the study.

It should be noted that after 1 year of the study, we did not observe any significant changes in the studied parameters in healthy subjects. For the clarity of the analyses, these results have not been presented.

Analysis of the factors affecting the variability of $\mathrm{Tf}$

Table 5 demonstrates only these correlations that were statistically significant at the beginning and after 1 year 


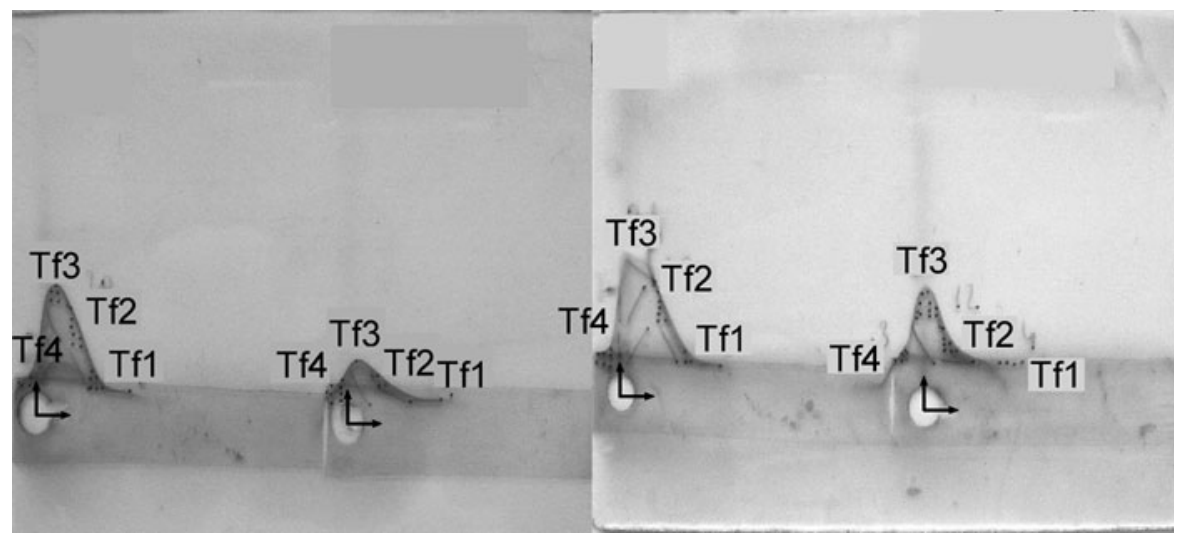

Fig. 4 Tf microheterogeneity patterns of sera of selected patients treated with maintenance haemodialysis analysed by the crossed affinoimmunoelectrophoresis according to BogHansen in the presence of Concanavalin A in the first- dimension gel (the horizontal pointer) and rabbit anti-human transferrin in the second-dimension gel (the perpendicular pointer)

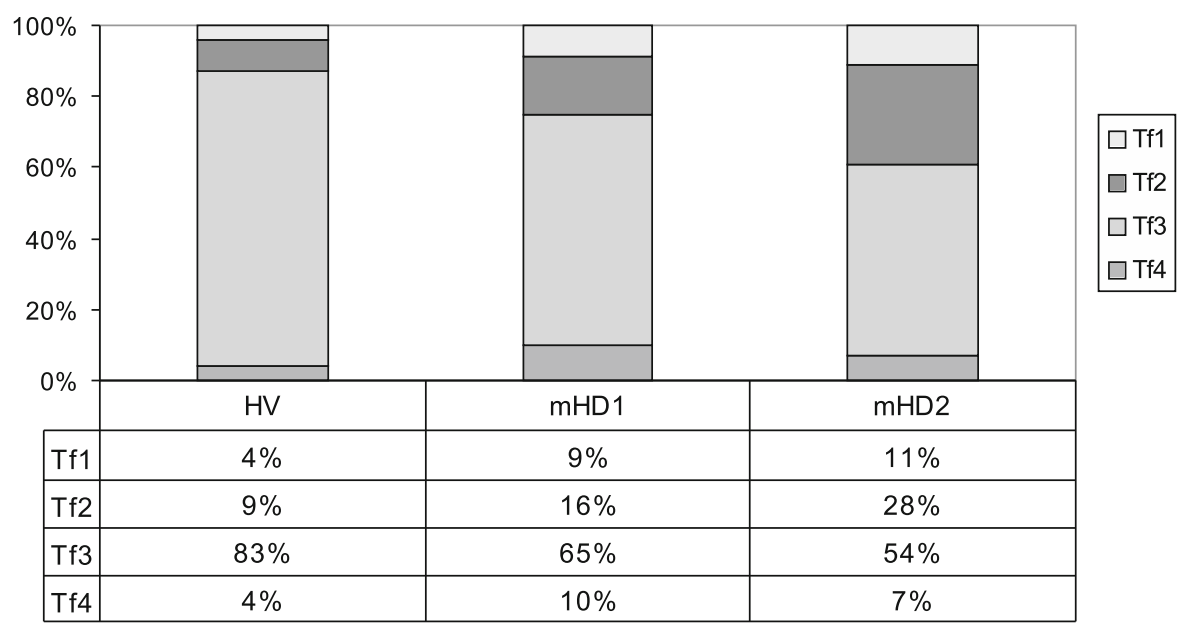

Fig. 5 Per cent distribution of Tf variants in the whole transferrin serum concentration in studied groups. Subjects were divided into patients treated with maintenance haemodialysis (mHD patients) and healthy volunteers (HV). In the

of the study. Significant inverse correlations, in both mHD1 and mHD2 groups, were found for the following associations: Tf3 and the duration of HD treatment, Tf3 and hsCRP and Tf3 and IL-6. Moreover, Tf3 variant showed significant positive correlations with HGB, $\mathrm{RBC}, \mathrm{Fe}$ and eGFR. No statistically significant correlations in both studied group of haemodialysed patients were observed between remaining Tf variants and these above-mentioned parameters.

Furthermore, we did not find any other correlations between Tf variants and age, sex, the existence of cardiovascular disease, hypertension, diabetes mellitus,
mHD group, there are two subgroups: mHD1 (patients at the beginning of the study) and mHD2 (the same group of patients after 12 months of the study)

WHR, BMI, selected liver metabolism parameters, TNF-alpha and rHuEPO and iron doses.

\section{Discussion}

Chronic kidney disease shares several features of the inflammatory state. One link between elevated levels of pro-inflammatory cytokines and poor treatment outcomes in CKD patients may be the presence of anaemia. Evidence suggest several mechanisms, like suppression of bone marrow erythropoiesis [26], 
Table 5 Statistically significant correlations found in haemodialysed patients

\begin{tabular}{lll}
\hline & Tf3 & \\
\cline { 2 - 3 } & mHD1 & mHD2 \\
\hline Duration of HD treatment & $r=-0.41$ & $r=-0.43$ \\
& $P=0.04$ & $P=0.033$ \\
eGFR & $r=0.88$ & $r=0.55$ \\
RBC & $P=0.0001$ & $P=0.004$ \\
HGB & $r=0.57$ & $r=0.55$ \\
Fe & $P=0.003$ & $P=0.004$ \\
& $r=0.4$ & $r=0.88$ \\
hsCRP & $P=0.004$ & $P=0.0001$ \\
& $r=0.55$ & $r=0.58$ \\
IL-6 & $P=0.004$ & $P=0.002$ \\
& $r=-0.46$ & $r=-0.047$ \\
& $P=0.019$ & $P=0.021$ \\
& $r=-0.176$ & $r=-0.186$ \\
& $P=0.041$ & $P=0.029$ \\
\hline
\end{tabular}

mHD1-patients at the beginning of study and mHD2patients after 1 year of the study

To calculate the correlations-Spearman's rank correlation coefficient was used

Tf3 variant of transferrin, $H D$ haemodialysis, $e G F R$ estimated glomerular filtration rate, $R B C$ red blood cells, $H G B$ haemoglobin, $\mathrm{Fe}$ serum iron, $h s C R P$ high sensitivity $\mathrm{C}$-reactive protein, IL-6 interleukin 6

Significant correlation when $P<0.05$

suppression of EPO production [27], increased intestinal bleeding [28] and modulation of iron metabolism [29, 30] by which inflammatory cytokines may affect the development of anaemia.

Our paper dealt with the issue related to iron deficiency in dialysis patients. There are three known main mechanisms that have been proposed to explain the high frequency of iron deficiency in these patients, in addition to the increased demand for iron and consequent storage in RBCs when erythropoiesis is stimulated with rHuEPO. These include abnormal iron absorption, external blood loss and functional iron deficiency [31]. The last mentioned disturbance, observed also in patients from our study, is present when the usual tests for iron deficiency in dialysis patients do not indicate absolute iron deficiency (ferritin of more than $100 \mathrm{ng} / \mathrm{ml}$; TAST of more than $20 \%$ ), but patients respond to additional iron administration with a rise in haematocrit at a stable rHuEPO dose or maintain a stable haematocrit with a lower
rHuEPO dose [32]. Patients with functional iron deficiency therefore have apparently insufficient available iron to keep up with the demands of the stimulated erythropoiesis that occur when rHuEPO is administered. In some patients, the inability to mobilise iron rapidly depends on the presence of reticulo-endothelial blockade, which may be the effect of the increased levels of cytokines [32]. In general, circulating cytokines may impair iron metabolism via several different mechanisms [33].

This persistent chronic inflammatory state may contribute to the changes of variability in Tf levels and hyporesponsiveness to proper treatment often seen in CKD patients. Modification in glycosylation of acute phase proteins (APPs), Tf being one of them (negative APPs), evoked by the changes in cytokine concentration, can be studied using a crossed immunoaffinity electrophoresis with lectins, e.g. Concanavalin A. This lectin strongly binds covalently to biantennary sugar chains and does not bind tri-, tetra- or more branched glycans, nor structures with bisecting $\mathrm{N}$-acetylglucosamine [34]. Therefore, protein molecules can be divided into ConA-reactive variants (with predominantly bi-antennary glycans) and ConA non-reactive variants, i.e. more branched. This mentioned electrophoresis allows the complete resolution of human $\mathrm{Tf}$ into variants. The number of four $\mathrm{Tf}$ variants found in this study agrees with this reported by Sobiesiak [35] and by Grzymisławski et al. [36]. On the other hand, according to Léger et al. [11], human serotransferrin was resolved only into three carbohydrate molecular variants, i.e. TfI (two triantennary glycans), TfII (one tri-antennary glycans and one bi-antennary glycans) and TfIII (two bi-antennary glycans), corresponding to Tf1, Tf2 and Tf3, respectively. The main difference in comparison with our results was the lack of $\mathrm{Tf} 4$.

Following is the description of the different variants, supplemented by information about their reaction with ConA during electrophoresis, together with a discussion of the obtained results.

The first Tf variant (Tf1) is not retarded in reaction with ConA, it means that it bears glycans that ConA cannot bind (tri or more antennary) or sugars incomplete or completely absent. It is known that this variant raises both in chronic and in acute stages of the diseases [35]. Our results did not confirm these findings. At the beginning of the study, we observed a slightly statistically significant decrease in the serum 
concentration of this variant, but at the end its concentration did not differ if compared to controls. Moreover, the differences between mHD1 and mHD2 were not significant, despite the fact that during the annual observation we noticed an increase in the inflammatory process in $\mathrm{mHD}$ patients. However, during the annual observation, the percentage content of this variant in the concentration of the total serum Tf increased in comparison with the results obtained at the start of the study and when we compared these results with controls. Further studies are required to know how important this variant is in iron transport.

The second variant (Tf2) reacts slightly with ConA, thus this variant should carry bi-antennary sugar chains. This could be a glycoform with two chains: one bi- and the other tri-antennary, the socalled 'pentasialo Tf' [35]. Tf2 may consist also of two isomeric three-antennary chains Noismith and Field [34]. During this study, we have observed that its serum concentration increased similarly to the percentage contents in the total Tf serum concentration. However, its serum concentration after 1 year of the study did not differ significantly in comparison with controls. As with the previous Tf, determining the significance of this variant in the transport of iron requires further research.

The Tf3 is the most dominant one. It is strongly retarded in electrophoresis with ConA; thus, it carries molecules with two bi-antennary sugar chains, the socalled tetrasialo Tf. Probably this variant is apotransferrin (apoTf) free for iron transport in the human organism [35, 37]. Normally, all of non-haem iron in the circulation is bound to holotransferrin (holoTf). Only about $30 \%$ of $\mathrm{Tf}$ binding sites are occupied, so that most of this protein is free of iron and is able to bind one or two $\mathrm{Fe}^{3+}$ ions in transferrins' $\mathrm{N}$ - and/or T-terminal ends $\left(\mathrm{Nt}\left(\mathrm{Fe}^{3+}\right)\right.$ holoTf or holoTf $\left(\mathrm{Fe}^{3+}\right)$ $\mathrm{Ct}$ or holoTf $\left.\left(\mathrm{Fe}^{3+}\right)_{2}\right)$. ApoTf competes with bacteria for the free iron ions to limit an infection and after the binding with the iron it is transformed into holoTf, so its concentration decreases. Indirect confirmation that Tf3 is probably apo-Tf, we found in the results of our research. Namely, in mHD patients we observed a reduction in UIBC, a parameter reflecting the level of Tf, which has not been used for the transport of iron, and this reduction correlated with the lowering Tf3. It should highlight, however, that a statistically significant relationship between mentioned parameters was detected only in patients from mHD2 group, so we did not put it into result set. Our results, both at the beginning and after 12 months of the observation, showed the lower concentration of serum Tf3 and the lower percentage content of Tf3 in the total Tf serum concentration, if compared to HV. It should be noted that after 1 year of the study these changes intensified. This is particularly important because this variant is likely the crucial one in the transport of iron, which was indirectly confirmed in the observed correlations, i.e. positive correlations between Tf3 and Fe, HGB, RBC, respectively. Furthermore, our results revealed that impaired renal function and the duration of the mHD treatment may have an influence on the serum concentration of this variant (positive correlations between these parameters). However, it seems that the main factor that influences Tf3 is the inflammatory process. Indirect proofs of this impact are the inverse correlations between the concentration of this variant and both hsCRP and IL-6.

The last variant, i.e. Tf4 variant, is the slowest one in a crossed immunoaffinity electrophoresis with ConA. Its mobility may be influenced by other heterogeneity factors like saturation with iron, the quantity of sialic acid or any changes in the structure of the protein [35]. During 1 year of our study, it was revealed that it is the serum concentration that decreased, similarly to its percentage content in the total $\mathrm{Tf}$ serum concentration. On the other hand, the almost double increase in percentage content of this variant in mHD group in comparison with HV group was found. This phenomenon is probably connected with intensive processes of the reconstruction of the $\mathrm{Tf}$ oligosaccharides chains observed during chronic inflammatory process, and therefore the increase in the quantity of $\mathrm{Tf}$ with defectible carbohydrates was observed in our study.

As was mentioned earlier, Tf, produced by the liver, belongs to APPs and modifications of its glycosylation, evoked mainly by the changes of circulating cytokines, may be important indicator of acute phase response. To make sure that the changes observed in our study are not due to factors whose impact is commonly known, i.e. liver dysfunction and nutritional status - we tried to rule out their influence on the results. On the other hand, we assessed only WHR, BMI and serum albumin levels, so we cannot with certainty exclude the impact of nutritional status on the observed changes, although the parameters examined, i.e. WHR, albumins, BMI, did not indicate that nutritional status could explain the obtained the results. 
The observed changes, i.e. the lower serum concentration of $\mathrm{Tf}$, the higher serum concentrations of ferritin, C-reactive protein and selected cytokines, i.e. IL-1, IL-6 and TNF-alpha found during this study confirmed the existence of the chronic inflammatory process in studied patients. Many chronic diseases, including end-stage renal disease, are associated with spuriously high serum ferritin levels notwithstanding low tissue iron levels, what we have also observed in our study. Historically, serum ferritin was thought to increase when intracellular ferritin levels were high, so that high serum levels of this protein were indicative of intracellular ferritin concentration or intracellular stores. Two lines of evidence challenge this thesis. First, intracellular ferritin appears to be a completely different protein species than circulating in serum. Second, it is also known to increase nonspecifically as an acute phase reactant [32].

To test whether the inflammatory process itself may induce changes observed during this examination, we conducted an additional analysis. For this purpose, we examined twice, at the beginning and after 1 year of the treatment, 10 haemodialysed patients without clinical and laboratory features of chronic inflammation. In this small group of patients, we found similar changes of the variability of $\mathrm{Tf}$ variants to those observed in the main groups of mHD patients. The only difference was the consistency of these changes, i.e. no statistically significant differences were revealed during the annual follow-up. Moreover, the per cent content of $\mathrm{Tf}$ variants in the whole serum $\mathrm{Tf}$ was similar to changes observed in mHD1 group. These results were not included into this work because of too small number of patients enrolled to the study group. We mention them to indicate that inflammation is an important factor that influences $\mathrm{Tf}$ variants, but it seems that it is not the only one.

Summarising, the inflammatory process in CKD has an influence on $\mathrm{Tf}$ and, in particular, on its variants. In our study, we observed that during 1 year of the haemodialysis treatment the serum concentration of the total $\mathrm{Tf}$ was constant, but lower if compared to controls, whereas the percentage of the individual Tf variants changed. It is worth to mention that alterations in the glycosylation profile of serum proteins may occur faster than in its general concentration, which could suggest peripheral binding of selected variants or further processing in peripheral blood or tissues. Synthesis and glycosylation are regulated distinctly [37]. Assessments of APPs may even be more reliable than the exemplary IL-6 serum concentration, as this cytokine is produced locally and quickly catabolised [35].

Pathogenesis of the anaemia associated with CKD and $\mathrm{mHD}$ is multi-factorial, and new factors are still investigated. The Tf heterogeneity changes may be one such additional new factors. Future research is needed to clarify this issue.

Acknowledgments This research has been partially supported by the Polish Ministry of Science and Higher Education grant No. N N 402209833.

Open Access This article is distributed under the terms of the Creative Commons Attribution Noncommercial License which permits any noncommercial use, distribution, and reproduction in any medium, provided the original author(s) and source are credited.

\section{References}

1. Formanowicz D, Sackmann A, Formanowicz P, Błażewicz J (2007) Petri net based model of the body iron homeostasis. J Biomed Inform 40:476-485

2. Sackmann A, Formanowicz D, Formanowicz P, Koch I, Blazewicz J (2007) An analysis of the Petri net based model of the human body iron homeostasis process. Comput Biol Chem 31:1-10

3. Formanowicz D, Sackmann A, Kozak A, Błażewicz J, Formanowicz P. Some aspects of anemia of chronic disorders modeled and analyzed by petri net based approach. Bioprocess Biosyst Eng. doi:10.1007/s00449-010-0507-6

4. Nuroko S (2006) Anemia in chronic kidney disease: causes, diagnosis, treatment. Cleve Clin J Med 73:289-297

5. Anderson BF, Baker HM, Norries GF, Rice DW, Baker EN (1989) Structure of human lactoferrin: crystallographic structure analysis and refinement at $2.8 \AA$ resolution. J Mol Biol 209:711-734

6. Arndt T (2001) Carbohydrate-deficient transferrin as a marker of chronic alcohol abuse: a critical review of preanalysis, analysis, and interpretation. Clin Chem 47:13-27

7. Giannetti AM, Snow PM, Zak O, Björkman PJ (2003) Mechanism for multiple ligand recognition by the human transferrin receptor. Plos Biol 1:341-350

8. Kamboh MI, Ferrell RE (1987) Human transferrin polymorphism. Rev Hum Hered 37:65-81

9. de Jong G, van Noort WL, van Eijk HG (1992) Carbohydrate analysis of transferrin subfractions isolated by preparative isoelectric focusing in immobilized $\mathrm{pH}$ gradients. Electrophoresis 13:225-228

10. Sobiesiak M (2002) Reactivity of human serum transferrin with two mannose-binding lectins: LCA and ConA. Cent Eur J Immunol 27:63-68

11. Léger D, Campion B, Decottigenies JP, Montreuil J, Spik G (1989) Physiological significance of the marked 
increased branching of the glycans of human serotransferrin during pregnancy. Biochem J 257:231-238

12. Wu Y, Sakamoto H, Kanenishi K, Tanaka S, Ueno M, Hata T (2004) Transferrin microheterogeneity in fetal blood. Biol Neonate 86:98-103

13. Stibler H, Borg S, Allgulander C (1979) Clinical significance of abnormal heterogeneity of transferrin in relation to alcohol consumption. Acta Med Scand 206:275-281

14. Rudolph JR, Regoeczi E, Chindemi PA, Southward S (1987) In: Proceedings of 8th international conference on proteins of iron transport and storage, Montebello, Quebec

15. van Dijk JP, van der Zande FG, Kroos MJ, Starreveld JS, van Eijk HG (1993) Number and affinity of transferrinreceptors at the placental microvillous plasma membrane of the guinea pig: influence of gestational age and degree of transferrin glycan chain complexity. J Dev Physiol 19:221-226

16. Pietrzak I, Formanowicz D, Sobieska M (2003) Transferrin microheterogeneity in patients with end stage renal disease treated by maintenance hemodialysis. J Am Soc Nephrol 14:532 (abstract)

17. Formanowicz D (2005) The meaning of the transferrin glycosylation and the concentration of the soluble transferrin receptor as factors having an influence on anemia in patients with end-stage renal disease treated by maintenance hemodialysis (in Polish). Doctoral dissertation, Poznań University of Medical Sciences, Poznań

18. Pietrzak I, Formanowicz D (2006) Transferrin microheterogeneity in diabetic and non-diabetic patients treated with maintenance haemodialysis. J Am Soc Nephrol 17: 480A (abstract)

19. Formanowicz D, Pietrzak I, Wiktorowicz K (2007) Mikroheterogenność transferyny u chorych leczonych powtarzaną hemodializą (in Polish). Przegl Lek 64:483-488

20. Formanowicz D, Pietrzak I (2007) Zmiany profilu glikozylacji transferyny u chorych w okresie schyłkowej niewydolności nerek leczonych powtarzaną hemodializą (in Polish). Diag Lab 43:321-330

21. The national Kidney foundation kidney disease outcomes quality initiative. http://www.kidney.org/professionals/kdoqi/

22. Levey AS, Greene T, Kusek J, Beck G (2000) A simplified equation to predict GFR from serum creatinine. J Am Soc Nephrol 11:155A (abstract)

23. Gotch FA, Sargent JA (1985) A mechanistic analysis of the National Cooperative Dialysis Study (NCDS). Kidney Int 28(3):526-534

24. Laurell CB (1973) Quantitative estimation of proteins by electrophoresis in agarose gel containing antibodies. Scand J Clin Investig 124:21-28
25. Bog-Hansen TC (1973) Crossed immuno-affinoelectrophoresis: a useful tool to predict the result of affinity chromatography. Anal Biochem 65:480-488

26. Schooley JC, Kullgren B, Allison AC (1987) Inhibition by interleukin-1 of the action of erythropoietin on erythroid precursors and its possible role in the pathogenesis of hypoplastic anaemias. Br J Haematol 67:11-17

27. Miller CB, Jones RJ, Piantadosi S, Abeloff MD, Spivak JL (1990) Decreased erythropoietin response in patients with the anemia of cancer. N Engl J Med 322:1689-1692

28. Jongen-Lavrencic M, Peeters HR, Rozemuller H, Rombouts WJ, Martens AC, Vreugdenhil G, Pillay M, Cox PH, Bijser M, Brutel G, Breedveld FC, Swaak AJ (1996) IL-6induced anaemia in rats: possible pathogenetic implications for anemia observed in chronic inflammations. Clin Exp Immunol 103:328-334

29. Kooistra MP, Niemantsverdriet EC, van Es A, Mol-Beermann NM, Struyvenberg A, Marx JJ (1998) Iron absorption in erythropoietin-treated haemodialysis patients: effects of iron availability, inflammation and aluminium. Nephrol Dial Transplant 13:82-88

30. Nemeth E, Tuttle MS, Powelson J, Vaughn MB, Donovan A, WcVey Ward D, Ganz T, Kaplan J (2004) Hepcidin regulates cellular iron efflux by binding to ferroportin and inducing its internalization. Science 306:2051-2053

31. Fishbane S, Maesaka JK (1997) Iron management in endstage renal disease. Am J Kidney Dis 29:319-333

32. Nissenson AR, Strobos J (1999) Iron deficiency in patients with renal failure. Kidney Int 55:18-21

33. de Francisco ALM, Stenvinkel P, Vaulont S (2009) Inflammation and its impact on anaemia in chronic kidney disease: from haemoglobin variability to hyporesponsiveness. NDT Plus 2(Suppl 1):18-26

34. Noismith JH, Field RA (1996) Structural basis of trimannoside recognition by concanavalin A. Glycobiology 271:972-976

35. Sobiesiak M (2003) Acute phase proteins in transplant patients-review. Transplantationsmedizin 15:25-30

36. Grzymisławski M, Derc K, Sobieska M, Wiktorowicz K (2006) Microheterogeneity of acute phase proteins in patients with ulcerative colitis. World J Gastroenterol 12:5191-5195

37. van Noort WL, de Jong G, van Eijk HG (1994) Purification of isotransferrins by concanavalin A sepharose chromatography and preparative isoelectric focusing. Eur J Clin Chem Clin Biochem 32:885-892 